\title{
A railway wheel evaluation under multiaxial loading conditions
}

\author{
Henrique SOARES ${ }^{1}$, Vitor ANES ${ }^{2}$, Manuel FREITAS ${ }^{3,4}$, Luis REIS $^{1,3, *}$ \\ ${ }^{1}$ Instituto Superior Técnico, Universidade de Lisboa, Av. Rovisco Pais, 1, 1049-001 Lisbon, Portugal \\ ${ }^{2}$ Instituto Superior de Engenharia de Lisboa, Instituto Politécnico de Lisboa, Rua Conselheiro Emídio \\ Navarro, 1, 19559-007, Lisbon, Portugal \\ ${ }^{3}$ IDMEC, Instituto Superior Técnico, Universidade de Lisboa, Av. Rovisco Pais, 1, 1049-001 Lisbon, \\ Portugal \\ ${ }^{4}$ Atlântica, University School, Fábrica da Pólvora, Barcarena, 2730-036 Barcarena, Portugal
}

\begin{abstract}
Railway mechanical components are subject to thousands of fatigue cycles. Fatigue damage and life assessment is still an open issue. Under service multiaxial loading conditions several challenges can arise. In this study an evaluation of a railway wheel material is performed, i.e. the material properties and the working conditions are taking into account and evaluated. Different mechanical tests are carried out, namely fatigue tests under uniaxial (LCF+HCF) and biaxial (HCF) conditions, applied to several specimens made from the railway wheel. Multiaxial fatigue models were considered regarding the fatigue life estimation. Moreover, fatigue crack plane measurements were compared with estimations from several critical plane models. The applied models provided very satisfactory results, regarding the fatigue life estimation and the initial crack initiation plane under the multiaxial loadings conditions.
\end{abstract}

\section{Introduction}

Fatigue studies are of the utmost importance for any system subjected to dynamic loadings. This type of failure is one of the most common and unpredictable and may happen in any range from low to very high number of cycles [1].

Therefore, acknowledging the material fatigue resistance provides a more reliable and secure mechanical system, structure or component. Several fatigue tests may be produced depending on the type of loading and on the number of cycles of interest. For the low cycle fatigue (LCF) and high cycle fatigue (HCF) regimes, conventional servo-hydraulic machines are usually used, where the rate of cycle application is not very high (below $50 \mathrm{~Hz}$ ) but easily applicable and controllable [2].

In general, when structural and machine components are working, they are subjected to multiaxial stress states mainly due to geometric form and/or complex loading. The lifetime estimation due to the fatigue of these components is extremely important [3], once it is related to safety operating conditions.

\footnotetext{
1 Corresponding author: * luis.g.reis@tecnico.ulisboa.pt
} 
Therefore, the railway wheels are designed to operating during a large lifetime, in general, around one million of kilometres before showing any problem. Afterwards, they are replaced in its regular conditions [4].

They always need to be replaced due to wear and almost never due to other type of faulty issue. However, due to different working conditions and events related to its manufacturing process, some defects can occur in its use, consequently forcing a reprofiling or scrap operation [5].

In this work, a railway wheel material is set under evaluation using conventional fatigue testing in order to evaluate its response on fatigue regime. The LCF and HCF regimes were studied using the servo-hydraulic machine with standardized specimens.

\section{Material and Methods}

The component in study is a worn-out railway wheel that should follow the EN_13262+A12009 standard [6]. The machine used in the multiaxial fatigue experiments was an Instron 8874 equipped with a $25 \mathrm{kN}$ and $100 \mathrm{~N} . \mathrm{m}$ load cell. All specimens were machined directly form the railway wheel from specific location as shown in Fig. 1. The specimen geometry and its dimensions are presented in Fig. 2.

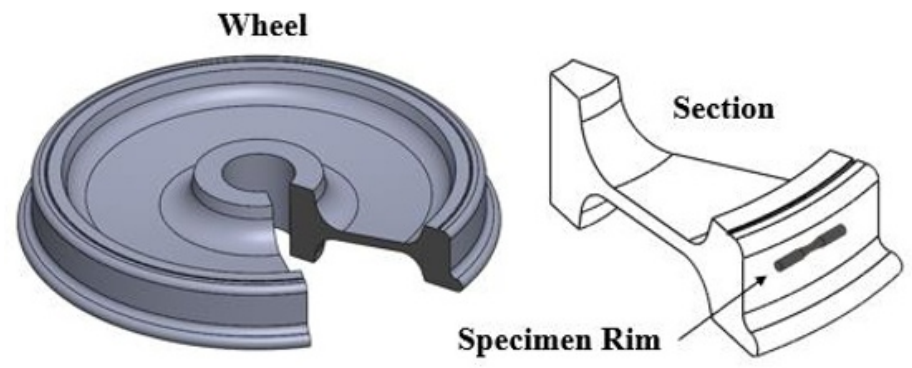

Fig. 1. Representation of the specimen's location on the railway wheel.

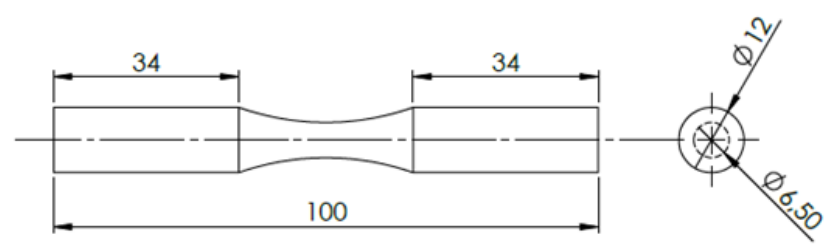

Fig. 2. HCF specimen that follows the ASTM E606 (2003) standard (mm) [7].

Four types of loadings were applied; Case 1 (pure shear loading), Case 2 (uniaxial loading), Case 3 (proportional loading), and Case 4 (non-proportional loading- $90^{\circ}$ phase shift). The corresponding loading paths are shown in Fig. 3. All tests were carried out in a servohydraulic testing system machine Instron model 8874 with a $5 \mathrm{~Hz}$ frequency at3 R=-1. Runouts were considered at 1E06 cycles. 


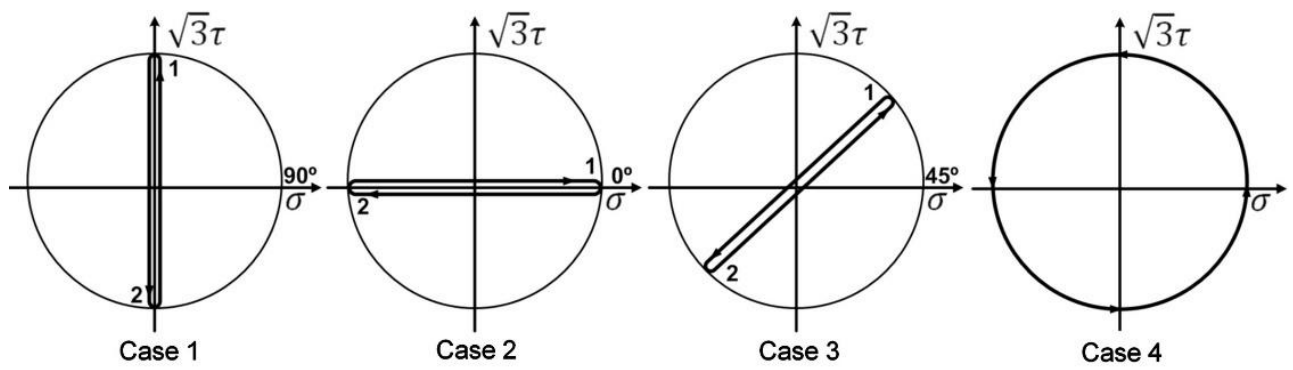

Fig. 3. Loading cases: Case 1 - Shear loading, Case 2 - uniaxial loading, Case 3 - proportional loading, Case 4 - out-of-phase loading.

\section{Theoretical approach}

\subsection{Critical plane models}

Critical plane models are suitable to estimate crack initiation planes. Most used criteria are the Brown-Miller, Findely, Fatemi \& Socie, Liu I, Liu II and SWT [8]. These models use a constant damage scale between normal and shear loads to estimate critical planes and estimate fatigue lives [9]. In this study, the influence of heat treatment in the crack initiation plane is analyzed by correlating the critical plane estimates with the experimental crack initiation planes.

\subsection{The stress scale concept}

Uniaxial SN curves are used in the design against fatigue failure of mechanical components. These curves are obtained in laboratory for a given material under push-pull or rotating bending loadings with $\mathrm{R}=-1$. In many practical cases, mechanical components are subject to multiaxial stress states, therefore the direct application of uniaxial SN curves to estimate fatigue strength is not possible, it is necessary to transform multiaxial stress states into an equivalent uniaxial stress state to do so. In most of multiaxial fatigue models this is made using a constant that transforms both normal and shear stress into the same stress space. In fact, this procedure is also made in static loading conditions too, for example, the von Mises equivalent stress, usually used in the design against yielding, is suitable to compare multiaxial stress states with yield stresses. This equivalent stress is a normal stress, where the shear stress component is transformed for the normal stress space using the factor 3, please see Eq. (1).

$$
\sigma_{v M}=\sqrt{\sigma^{2}+3 \tau^{2}}
$$

This transformation allows the summation of normal and shear stress, because they are in the same stress space, this means that the direct summation of normal and shear stresses, cannot be made. This can be illustrated considering the relation between normal and shear yield stresses. Generally, normal yield stress is considerably higher than the shear yield stress, therefore, the stress level in shear to yield a given material is lower than the one needed using normal stresses. Thus, normal and shear stresses have different scales because the same result (yielding in this context) is obtained with different stress levels.

Considering a uniaxial loading in shear, the von Mises stress is obtained as follows in Eq. (2). The equivalent von Mises stress is a normal stress, therefore the shear stress in Eq. (2) is transformed into the normal stress space using a stress scale factor of $\sqrt{3}$. 


$$
\sigma_{v M}=\sqrt{3} \tau
$$

The same reasoning can be made considering the shear stress space. In this case, Eq. (2) is rearranged into Eq. (3). Therefore, one can obtain a von Mises equivalent stress in the shear stress space, please see Eq. (4).

$$
\begin{gathered}
\tau=\frac{\sigma_{v M}}{\sqrt{3}}=\frac{\sigma}{\sqrt{3}} \\
\tau_{v M}=\sqrt{\left(\frac{\sigma}{\sqrt{3}}\right)^{2}+\tau^{2}}
\end{gathered}
$$

The von Mises equivalent stress transformation from normal stress space into shear stress space allows the correlation between a static multiaxial stress state with a shear yield stress.

The stress scale concept can be also found in cyclic response of materials. For example, the uniaxial normal SN curve has a positive offset relatively to the uniaxial shear stress SN curve. This means that the normal fatigue limit is higher than the shear one. Considering that the stress amplitude is strictly related with fatigue damage, one can conclude that the uniaxial shear loading is more damaging than the normal one. Therefore, also in fatigue there is a scale factor between normal and shear stresses. This is particularly important, because to estimate multiaxial fatigue strength in mechanical design one need to use a uniaxial SN curve which is normally set in the normal stress space.

Several multiaxial fatigue models consider the stress scale between normal and shear stresses using a constant, like the one used in the von Mises equivalent stress, usually a ratio of normal fatigue limit to shear fatigue limit. However, recent studies in literature have shown that the stress scale between stress spaces is dependent on the stress levels, loading type and material $[10,11]$. The SSF equivalent stress is one example in which these aspects are taken into account, this equivalent stress is defined in the shear stress space and has a damage map that updates the stress scale (or damage scale) according to the stress level and loading type. Despite, being primarily defined in the shear stress space, the SSF can be also defined in the normal stress space in order to be possible use uniaxial normal SN curves to estimate fatigue strength of multiaxial loadings. Eq. (5) represents the equivalent SSF shear stress in the shear stress space $[10,11]$.

$$
\tau_{\text {eqv }}=\tau_{a}+\operatorname{ssf}\left(\lambda, \sigma_{a}\right) \cdot \sigma_{a}
$$

where $\operatorname{ssf}\left(\lambda, \sigma_{a}\right)$ is a two variable $5^{\text {th }}$ order polynomial that represents the SSF damage map for the shear stress space, please see Eq. (6) [10, 12, 13].

$$
\operatorname{ssf}\left(\sigma_{a}, \lambda\right)=a+b \cdot \sigma_{a}+c \cdot \sigma_{a}{ }^{2}+d \cdot \sigma_{a}{ }^{3}+f \cdot \lambda^{2}+g \cdot \lambda^{3}+h \cdot \lambda^{4}+i \cdot \lambda^{5}
$$

where $\sigma_{a}$ represents the loading normal stress component and aims to account for the loading stress level and $\lambda$ represents the ratio of shear stress amplitude to normal stress amplitude and aims to account for the loading type. The variables $a$ to $i$ are the polynomial constants obtained from experiments. These variables vary accordingly to the material type $[11,12]$.

To quantify the relative damage between proportional and non-proportional loading paths, Anes et al. [13] proposed the Y parameter, a non-proportional sensitivity parameter determined based on proportional and non-proportional loading tests. Eq. (7) shows the SSF equivalent shear stress updated to non-proportional loading conditions using the Y parameter.

$$
\tau_{e q v}=Y *\left(\tau_{a}+\operatorname{ssf}\left(\lambda, \sigma_{a}\right) \cdot \sigma_{a}\right)
$$


Figure 4 depicts the Y definition, where axial and shear SN curve components are related in order to estimate non-proportional fatigue damage based on the SSF proportional damage map represented by Eq. (6).

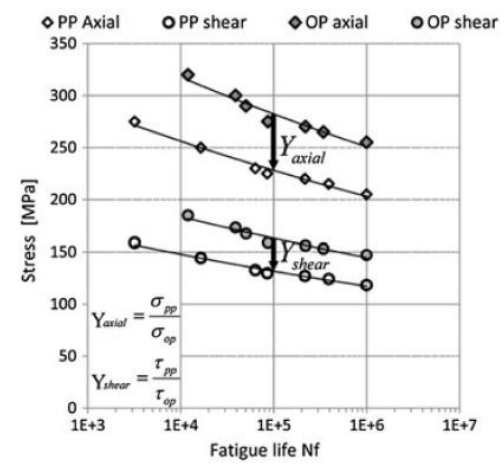

Fig. 4. Y parameter definition [13].

\section{Results and discussion}

Table 1 shows the stress scale factor variation for the four loading cases presented in Fig. 3 using the methodology proposed in [10]. These results show the effect of the loading path and stress level on the material fatigue strength. These effects are captured by the SSF variation with the material fatigue strength where the SSF varies accordingly to the number of loading cycles until failure. This behavior is in accordance with other materials tested by the present authors [12].

In the last column of Table 1, it is shown the $\mathrm{Y}$ factor for Case 4 . $\mathrm{Y}$ factor is a parameter that allows to take into account the material response to non-proportionality and has, as reference, the respective proportional loading case without phase shift (without non-proportionality).

In this sense, the results presented in column 5 of Table 1 represents a correction to the SSF equivalent stress to account the material response to non-proportionality. Analyzing this column one can concluded that for this material loading Case 4 causes less damage than Case 3 , especially for higher stress levels. Observing the last row of Table 1, where we get a $Y=1$, one can conclude that the damage caused by non-proportionality is very low, meaning that the SSF equivalent stress can be directly used to estimated fatigue strength of nonproportional loadings beyond the SN finite life region.

Table 1. Stress scale factors and Y factor for the material in study.

\begin{tabular}{|l|c|c|c|c|}
\cline { 2 - 5 } \multicolumn{1}{c|}{} & \multicolumn{3}{c|}{$s s f\left(\lambda, \sigma_{a}\right)$} & Y \\
\hline \multicolumn{1}{c|}{$\mathbf{N}_{\mathbf{f}}$} & Case 1 & Case 2 & Case 3 & Case 4 \\
\hline 1000 & 0,86 & 1 & 0,45 & 0,85 \\
\hline 10000 & 0,84 & 1 & 0,44 & 0,89 \\
\hline 100000 & 0,83 & 1 & 0,43 & 0,95 \\
\hline 1000000 & 0,81 & 1 & 0,42 & 1,00 \\
\hline
\end{tabular}


Fig. (s) 5 to 6 show the experimental crack initiation planes results for loading Cases 3 and 4 , in several tested specimens with different stress intensity levels. The results show that the crack initiation planes initiate predominantly around zero degrees in both loading Cases, proportional (Case 3) and non-proportional (Case 4), but crack initiation planes around $22^{\circ}$ were also achieved, which could be explained due to different stress intensity levels applied in the different specimens.

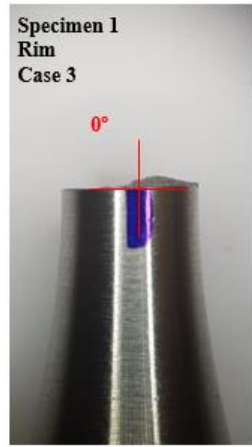

(A)

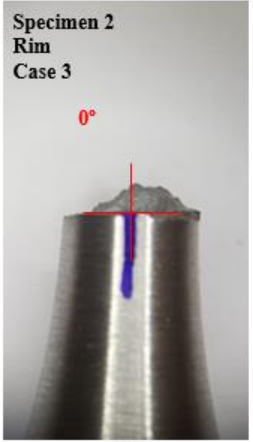

(B)

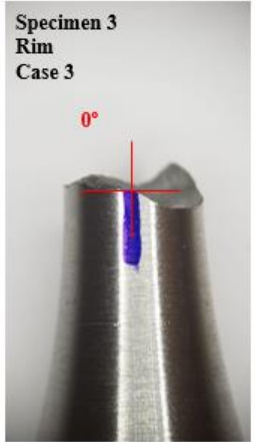

(C)

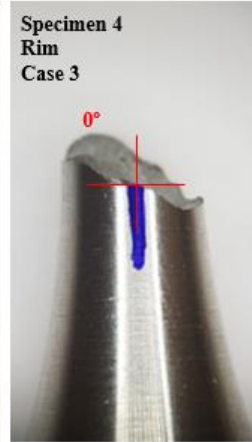

(D)

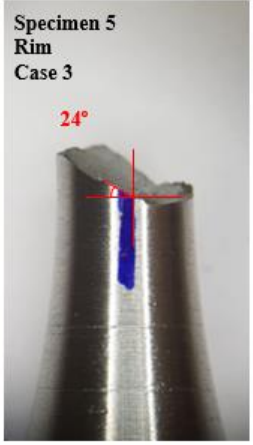

(E)

Fig. 5. Crack initiation planes measured for loading Case 3 .

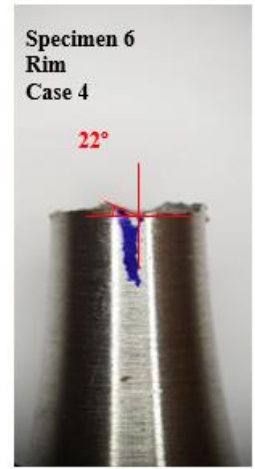

(A)

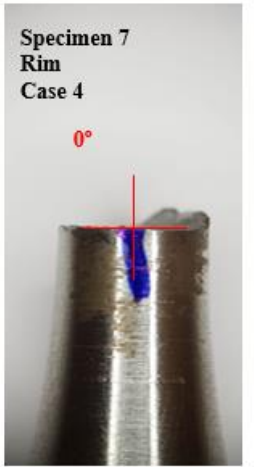

(B)

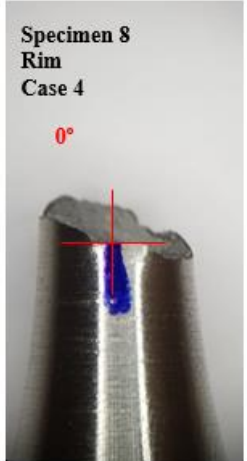

(C)

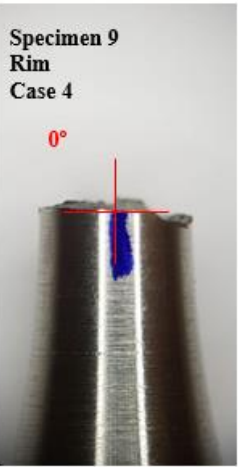

(D)

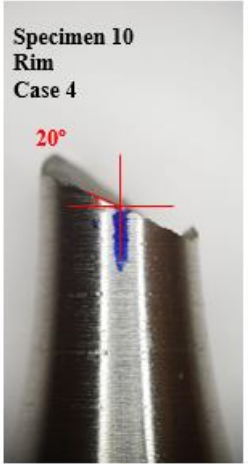

(E)

Fig. 6. Crack initiation planes measured for loading Case 4.

Table 2 shows the critical plane models estimates for loading Cases 3 and 4 . Based on Table 2 one can conclude that the critical plane estimates that better follows the crack initiation planes measured in the tested specimens are the SWT and LIU I models. These models are strongly based on axial loadings, which indicates that the tested material may be prone to damage caused by axial loadings.

Table 2. Critical plane estimates.

\begin{tabular}{|l|c|c|}
\cline { 2 - 3 } \multicolumn{1}{c|}{} & Case 3 & Case 4 \\
\hline Findley & $41^{\circ}$ & $41^{\circ}$ \\
\hline Brown-Miller & $-37^{\circ}$ & $-37^{\circ}$ \\
\hline$F-S$ & $39^{\circ}$ & $39^{\circ}$ \\
\hline$S-W-T$ & $0^{\circ}$ & $0^{\circ}$ \\
\hline Liu I & $0^{\circ}$ & $0^{\circ}$ \\
\hline Liu II & $\pm 45^{\circ}$ & $\pm 45^{\circ}$ \\
\hline
\end{tabular}


Fig. 7 (A) and (B) shows the damage parameter evolution for the SWT and LIU I models, respectively, where the maximum damage parameter identifies the crack initiation plane at theta equal to zero degrees.

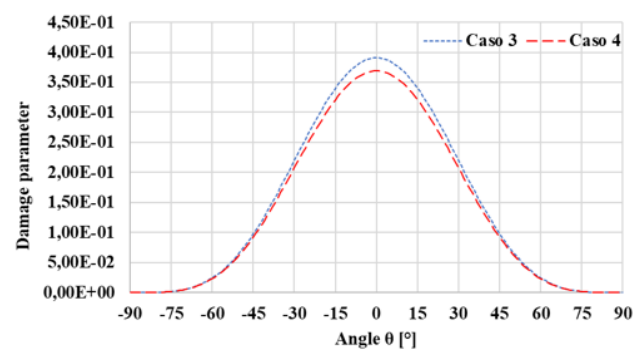

(A)

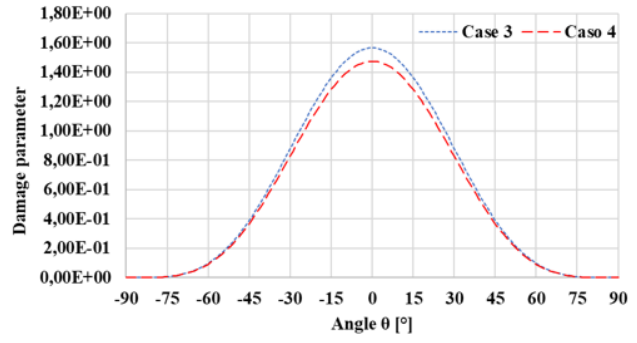

(B)

Fig. 7. Damage parameter evolution models: (A) SWT, (B) LIU I.

\section{Conclusions}

In this paper, the material of a used railway wheel was tested in lab in order to study its fatigue behavior under different loading paths. Several uniaxial and biaxial loading tests were performed under different stress levels, moreover, the respective crack initiation planes were identified and analyzed. Results show that the damage scale between axial and shear stresses needs to be taken into account when equivalent parameters are used. This must be done using a function instead of a constant as usually appears in many multi-axial fatigue models. Moreover, the non-proportional effect as a result of phase shift between axial and shear loading shows, for this material, to be less damaging than the in phase loading conditions. This result is captured by the $\mathrm{Y}$ factor that updates the axial and shear loading components of non-proportional loadings to match the correspondent proportional loading case, however, for low stress levels the differences between proportional and non-proportional can be neglected since the $\mathrm{Y}$ factor is equal to 1 .

The crack initiation study indicates that the experimental crack initiation plane is predominantly zero in both loading cases, and consequently the critical plane models that better predict the experimental results are the SWT and LIUI. These two models predominantly capture the influence of the axial damage of a biaxial loading, therefore the results achieved in loading Cases 3 and 4 may indicate that the tested railway material may be prone to initiate the damage primarily due to axial loadings.

This work was supported by FCT, through IDMEC, under LAETA, project UID/EMS/50022/2019. This work was also supported by Progama Ciência sem Fronteiras - CsF - BRASIL, project 9999.013107/2013-05.

\section{References}

1. C. Bathias, There is no infinite fatigue life in metallic materials, Fatigue Fract. Eng. Mater. Struct., 22, no. 7, 559-565, (1999).

2. H. Soares, P. Costa, M. Freitas, and L. Reis, Fatigue life assessment of a railway wheel material under HCF and VHCF conditions, MATEC Web Conf., 165, 09003, (2018). 
3. L. Reis, Comportamento Mecânico de Aços em Fadiga Multiaxial a Amplitude de Carga Constante e Síncrona, Instituto Superior Técnico, 2004.

4. H. Soares, T. Zucarelli, M. Vieira, M. Freitas, and L. Reis, Experimental characterization of the mechanical properties of railway wheels manufactured using class B material, Procedia Struct. Integr., 1, 265-272, (2016).

5. T. Zucarelli, L. Moreira Filho, H. Soares, M. Vieira, and L. Reis, Experimental characterization of the mechanical properties of railway wheels manufactured using class C material, Theor. Appl. Fract. Mech., 85, 134-139, (2016).

6. European Committee for Standardization, NF EN 13262+A1 - Railway applications - Wheelsets and bogies - Wheels - Product requirements, (2009).

7. ASTM International, Standard Test Method for Strain-Controlled Fatigue Testing, Annu. B. ASTM Stand., 96, 1-16, (2003).

8. D. Socie and G. Marquis, Multiaxial Fatigue, Society of Automotive Engineers, Inc. Warrendale, $\mathrm{Pa}$, 484, (2000).

9. H. Soares, V. Anes, M. Freitas, and L. Reis, Fatigue life of a railway wheel under uniaxial and multiaxial loadings, Procedia Struct. Integr., 13, 1786-1791, (2018).

10. V. Anes, L. Reis, B. Li, M. Fonte, and M. de Freitas, New approach for analysis of complex multiaxial loading paths, Int. J. Fatigue, 62, 21-33, (2014).

11. V. Anes, M. de Freitas, and L. Reis, The damage scale concept and the critical plane approach, Fatigue Fract. Eng. Mater. Struct., 40, no. 8, 1240-1250, (2017).

12. V. Anes, L. Reis, B. Li, and M. de Freitas, New cycle counting method for multiaxial fatigue, Int. J. Fatigue, 67, 78-94, (2014).

13. V. Anes, L. Reis, B. Li, and M. de Freitas, New approach to evaluate nonproportionality in multiaxial loading conditions, Fatigue Fract. Eng. Mater. Struct., 37, no. 12, 1338-1354, (2014). 